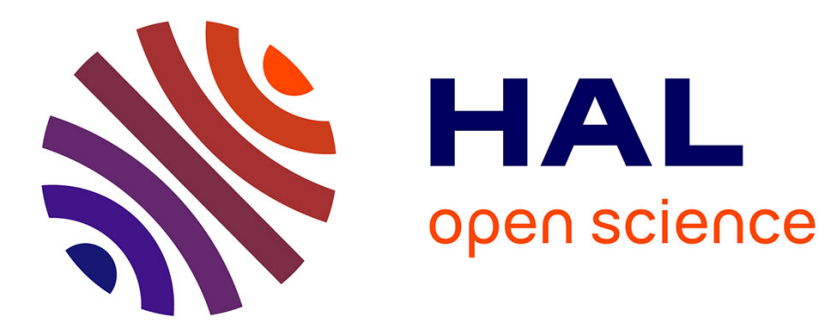

\title{
Dual-tuned birdcage-like coil based on metasurfaces
}

Nikulin Anton, Abdelwaheb Ourir, Julien de Rosny, Stanislav Glybovski, Benoît Larrat, Frank Kober, Redha Abdeddaim

\section{To cite this version:}

Nikulin Anton, Abdelwaheb Ourir, Julien de Rosny, Stanislav Glybovski, Benoît Larrat, et al.. Dualtuned birdcage-like coil based on metasurfaces. 2018 Days on Diffraction (DD), Jun 2018, St. Petersburg, Russia. pp.230-234, 10.1109/DD.2018.8553346 . hal-02506144

\section{HAL Id: hal-02506144 \\ https://hal.science/hal-02506144}

Submitted on 12 Mar 2020

HAL is a multi-disciplinary open access archive for the deposit and dissemination of scientific research documents, whether they are published or not. The documents may come from teaching and research institutions in France or abroad, or from public or private research centers.
L'archive ouverte pluridisciplinaire HAL, est destinée au dépôt et à la diffusion de documents scientifiques de niveau recherche, publiés ou non, émanant des établissements d'enseignement et de recherche français ou étrangers, des laboratoires publics ou privés. 


\title{
Dual-Tuned Birdcage-like Coil based on Metasurfaces
}

\author{
Nikulin Anton, Abdelwaheb Ourir, Julien de Rosny \\ Institut Langevin, ESPCI, PSL, CNRS UMR 7587, 75005 Paris, France; \\ e-mail: anton.nikulin@espci.fr \\ Stanislav Glybovski \\ ITMO University, St. Petersburg 197101, Russia \\ Benoit Larrat \\ DRF/I2BM/Neurospin/UNIRS, 91191 Gif-sur-Yvette Cedex, France \\ Frank Kober \\ Aix-Marseille Universite, CNRS, CRMBM, UMR 7339, Marseille, France \\ Redha Abdeddaim \\ Aix Marseille University, CNRS, Centrale Marseille, Institut Fresnel, 13013 Marseille, France
}

\begin{abstract}
We propose the dual-tuned volume coil for magnetic resonance imaging at two nuclei. This coil is based on the combination of two independent metasurfaces that can be used simultaneously for fluorine and hydrogen ${ }^{19} \mathrm{~F} /{ }^{1} \mathrm{H}$ imaging at 7 Tesla for small animals. Each metasurface is made of non-magnetic metallic rods and structural capacities of etched metal strips. The geometry is chosen so that two modes supported by the two metasurfaces resonate at the required frequencies $282.6 / 300.1 \mathrm{MHz}$. The two metasurfaces can be tuned independently by mechanically adjusting the length of the two sets of wires. We have numerically studied the concept of the proposed dual-tuned coil.
\end{abstract}

\section{INTRODUCTION}

Nowadays, Magnetic Resonance imagining is an essential apparatus in biology and medicine. This technique allows to provide $3 \mathrm{D}$ in-vivo anatomical images based on Larmor precession of the hydrogen nuclei $\left({ }^{1} H\right)$ under a strong static magnetic field. Complementary information can be obtained by probing nuclear magnetic resonance signals of spins of other nuclei. For instance fluorine-19 $\left({ }^{19} \mathrm{~F}\right)$ is used as a component of several contrast agents [1] allowing an accurate monitoring and tracking of injected cells into a body [1]. However, due to weak concentration of fluorine, ultrahigh-field MRI scanners are required to reach a sufficient signal to noise ratio (SNR). Moreover, as the Larmor frequency (i.e. the frequency of the nuclear magnetic resonance of spins) for the same magnetic field strengths depends on the nuclei type, dual-nuclei imaging requires a dual-tuned coil.

Recently it has been shown that hybridized resonators can improve the radio frequency ( $\mathrm{RF}$ ) field homogeneity and signal-to-noise ratio in the case of a single-nuclei application at $7 \mathrm{~T}$ [2]. Also, it has been proposed to excite two different modes of two hybridized wires structure with an electrically small loop antenna connected to MRI scanner only with one feeding channel [3]. A similar structure has been proposed later for the realization of a surface coil that operates at two independent frequencies providing independent tuning and matching [4].

In this paper, we propose a volume receive/transmit MRI antenna based on two independent metasurfaces that can be used simultaneously for fluorine and hydrogen ${ }^{1} \mathrm{H} /{ }^{19} \mathrm{~F}$ imaging of a small animal at 7 Tesla. The proposed dual-tuned coil can be tuned independently to the desired Larmor frequencies by adjusting the length of two sets of periodic telescopic wires located on cylindrical surfaces surrounding a subject to be scanned. This approach of tuning avoids the use of additional electronic elements and insertion losses of the latter. Thus, performance of the coil (i.e. quality of images) can be increased, meantime the price of manufacturing can be reduced. Our design is quite different to classical dual-tuned birdcages working as a combination of low-pass and high-pass birdcages for high-field MRI applications [5]. Such dual-tuned birdcages work well only when the two corresponding Larmor frequencies are considerably different, for instance ${ }^{1} H /{ }^{31} P$. [5]. 


\section{Coll Design}

The considered coil consists of two intricate 1D metasurfaces where the last unit cell is connected to the first one, forming a cylinder. The first metasurface behaves as a left-handed transmission line (LHTL). Actually, this structure corresponds to a high-pass birdcage structure proposed by Cecil Hayes in 1985 [6]. A conventional birdcage is very convenient for single-nuclei imaging because its fundamental mode provides homogeneous magnetic field inside the whole sample. In this work, we propose to use such a LHTL called birdcagelike metasurface (see Fig. 1a) for fluorine imaging. It consists of 6 legs (i.e. thin conductors directed along the cylindrical axis). The legs are repeated with the angular period $60^{\circ}$ around a $40 \mathrm{~mm}$-radius circumference of the cylinder. Each leg is made of two brass tubes with radius $1 \mathrm{~mm}$ and $0.75 \mathrm{~mm}$ to obtain a telescopic conductor with a mechanically adjustable length. On one side of the structure, the legs are interconnected by capacitors[7] and are short circuited to each other on the other side. The capacitors are etched on low-loss dielectric substrate Rogers $4003(\epsilon=3.38$ and $\delta=0.0021)$ with thickness $0.508 \mathrm{~mm}$. The part of the coil that is sensitive to the response of protons at $300.1 \mathrm{MHz}$ consists of an array of split ring resonators (SRRs), namely 6 split rectangular loops distributed along a circle of radius $30 \mathrm{~mm}$. The shape of the SRRs is rectangular with dimensions of $97 \mathrm{~mm}$ by 10 mm. The coupling between SRRs has been studied previously $[8,9]$. The relative orientation between SRRs changes the mutual inductance between them. It is positive when they are parallel or negative if coplanar [8]. As in the previous metasurface each SRR is telescopic, i.e., made of the assembly of $2 \mathrm{~mm}$ tube and $1.5 \mathrm{~mm}$ one. At one end, two wires are connected through a distributed capacity which is made of copper traces printed on a low-loss substrate (Rogers 4003). SRRs generate homogenous magnetic field is at the lowest frequency among the modes of the same metasurface. Schematic view of the split-ring resonators is shown in Fig. 1b.

\section{Numerical Simulations}

First, we test numerically the two structures separately using commercial software for $3 \mathrm{~d}$ electromagnetic simulations CST Microwave Studio 2018 (Frequency domain solver). All wires were placed along a) $50 \mathrm{Ohm}$ port 1 Birdcage-like metasurface

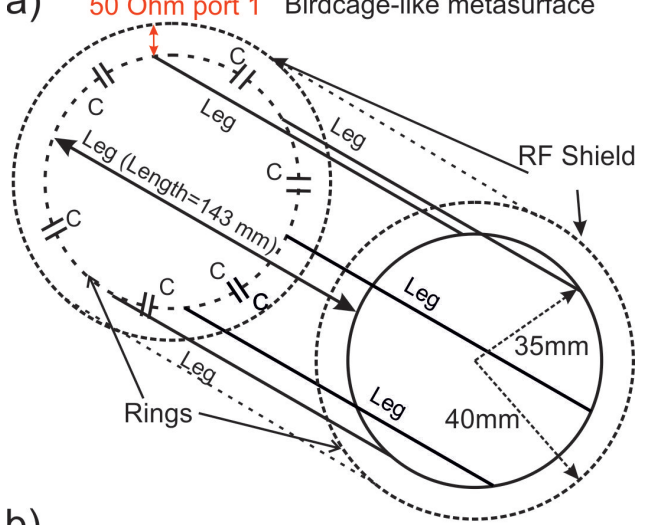

b) Metasurface of SRRs

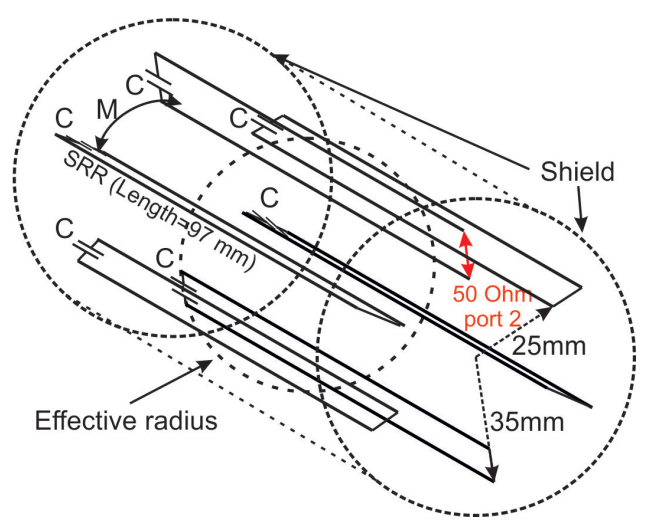

Figure 1: Schematic view of two metasurfaces comprising the proposed coil: (a) the metasurface of wires (the birdcage-like metasurface); (b) the metasurface of coupled split-ring resonators (the metasurface of SRRs).

$Z$-axis, axis of an MRI bore. Both metasurfacebased coils have been shielded by a cylindrical PEC with diameter $80 \mathrm{~mm}$. The $50 \mathrm{Ohm}$ excitation port for the birdcage-like metasurface is connected between one leg and shield (port 1). To excite the array of SRRs, we use a $50 \mathrm{Ohm}$ port connected in the gap of one SRRs (port 2). Both structures have been loaded by a cylindrical homogeneous phantom made of $60 \% 2-2$-trifluoroethanol and $40 \%$ water $(\epsilon=39$ and $\delta=0.06)$. Its radius and length are equal to $12.5 \mathrm{~mm}$ and $75 \mathrm{~mm}$, respectively. These values are related to typical small animal's sizes. Further, region of interest (ROI) corresponds to the phantom. All structures have been adjusted to work at desired frequencies of fluorine and hydrogen at 7 Tesla $(282.6 / 300.1 \mathrm{MHz})$ by adjusting the length of telescopic wires. The adjusted values are $143 \mathrm{~mm}$ and $97 \mathrm{~mm}$, respectively. Also, the 

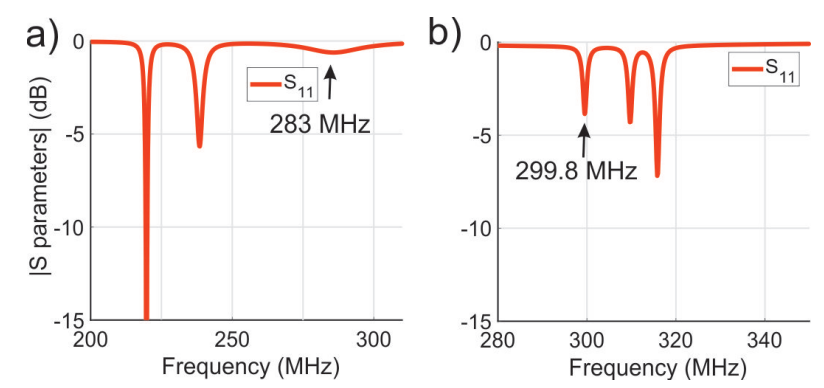

Figure 2: S11 (reflection coefficient) of simulated structures loaded by the phantom: (a) the birdcage-like metasurface; (b) the metasurface of SRRs.

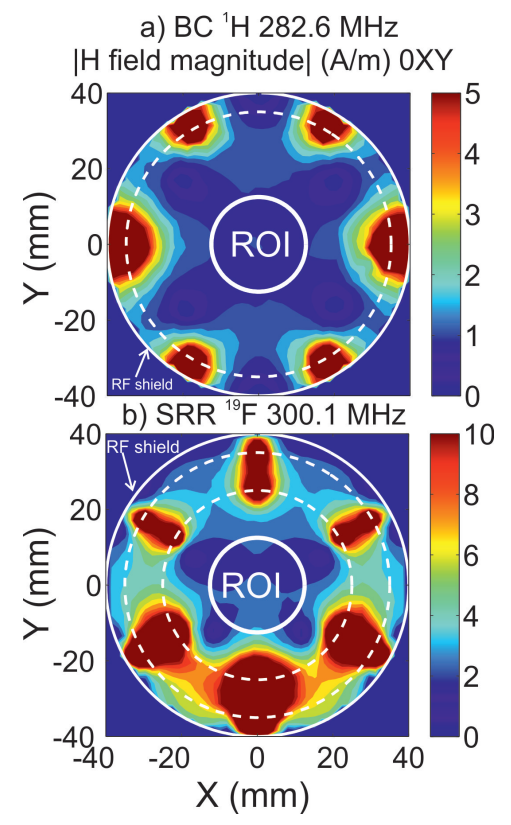

Figure 3: Amplitude of magnetic field inside the coil in central transverse plane $(z=0)$ : (a) mode of the birdcage-like metasurface at $282.6 \mathrm{MHz}$; (b) mode of the metasurface of SRRs at $300.1 \mathrm{MHz}$. The ROI is related to the cylindrical phantom used in the simulation.

overlapping between two parallel metallic plates of the connecting the wires at their ends has been adjusted to achieve preliminary frequencies tuning of both structures. Parameters of the structures are shown in Fig. 1a and b. respectively. S-parameters and magnetic field patterns are presented in Fig. 2 and Fig. 3 respectively. At both frequencies we are exciting the fundamental mode of the structures generating homogeneous magnetic field inside the coil, i.e., inside the region of interest (ROI).

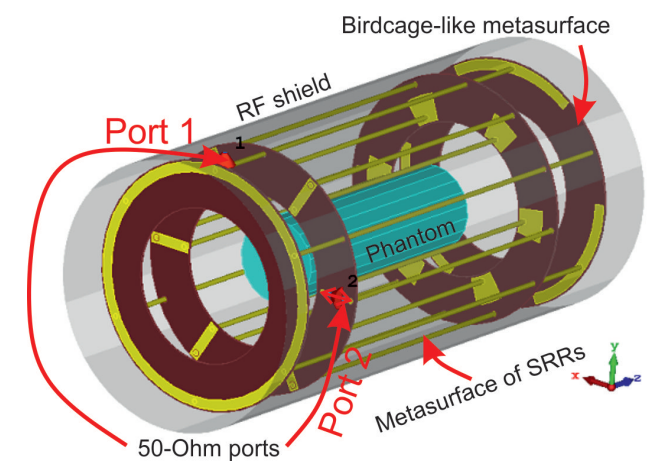

Figure 4: General view of the dual-tuned coil loaded by the phantom.

Next, both metasurfaces are combined into the same coil to work simultaneously at the two frequencies. Here, the metasurface of SRR is closed to external surface of the phantom, meantime the radius of the birdcage-like metasurface is equal to outer radius of the metasurface of SRR. Dimensions of both metasurfaces limited by the RF shield that can not be bigger then $80 \mathrm{~mm}$ due to the size of MRI bore. Also, decreasing of the radius of birdcage-like metasurface increase the mutual coupling between two metasurfaces. The final geometry of the dualtuned coil is presented in Fig. 4. The final setup including the RF shield and the phantom is not changed. In case of this combined coil all its dimensions coincide with ones of the two independently optimized single-tuned metasurfaces (see Fig. 1). In the Fig. 5 the S-parameters of combined structures are presented. Both structures seems to be not very sensitive to each other and their frequency shift after combining is negligible due to orthogonality of the modes. The slight variation of the resonant frequencies can be compensated by changing the length of telescopic wires.

As it was previously mentioned, the proposed coil maintains independent tunability of the resonant frequencies. In this case it is possible to reduce the number of elements of tuning and matching circuits and keep only one capacitor for each channel only for matching. In this case SNR of the images of the sample can be increased. S - parameters of independent tuning study are presented in Fig. 6. Initial S - parameters of the coil are presented in Fig. 6a. If the length of the metasurface of SRRs is modified, it affects mainly $S_{22}$ (Fig. 6b). Here, changing of $S_{11}$ can be neglected. In the opposite case (Fig. 6c) when the length of SRRs is initial and the length of the birdcage-like metasurface is modified it affects 


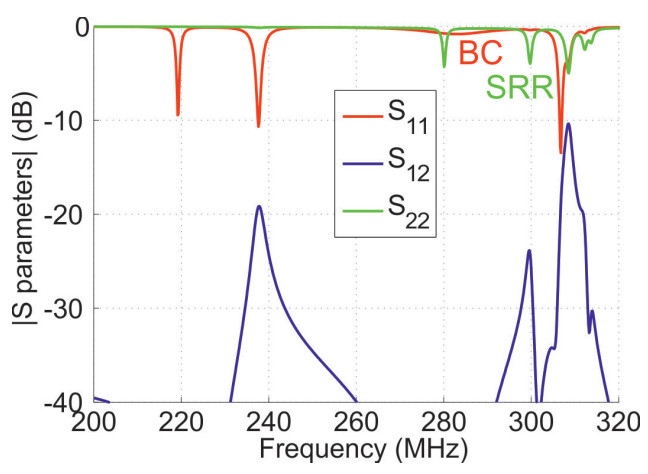

Figure 5: S-parameters of the combined dualtuned coil loaded by the phantom.

mainly $S_{11}$. Finally the dual-tuned coil tuned for the two desired Larmor frequencies of ${ }^{1} H$ and ${ }^{19} F$ is obtained. Contrary to conventional coils that require a matching/tuning circuit, here only a matching element, i.e. one tunable capacitor per channel is required.

\section{EFFICIENCY OF THE COIL}

To analyze the efficiency of the coil we have calculated $B_{1}^{+}$(clockwise circularly polarized) magnetic field distribution and have plotted it inside the ROI. In this case, the coil has been matched by capacitors and tuned by length adjustment for desired frequencies. To achieve a good matching level at 282.6/300.1 MHz, we use two tunable capacitors. The values of them are $2.55 \mathrm{pF}$ and $14.07 \mathrm{pF}$ for the birdcage-like metasurface and for the metasurface of SRRs structures, respectively. Ohmic losses in these capacitors were taken into account $(0.35 \mathrm{Ohm}$ and $0.15 \mathrm{Ohm}$ series resistance respectively). These two values are deduced from the quality factor of commercially available capacitors which is typically equal to 1000 in this frequency range and for these capacitance values. In this case, the reflection coefficient $\left|S_{11}\right|$ and $\left|S_{22}\right|$ are less than $-10 \mathrm{~dB}$. The level of parasitic transmission coefficient between the two ports is low. Indeed, $\left|S_{12}\right|$ is smaller than $-15 \mathrm{~dB}$. Calculated field pattern inside the phantom are presented in Fig. 7a for birdcage-like metasurface and in Fig. 7b for SRRs. In case of fluorine $(282.6 \mathrm{MHz})$ a very good homogeneity of $B_{1}^{+}$ field inside the region of interest is obtained (the birdcage-like metasurface). Indeed, the standard deviation of the field is equal to $0.04 \mu \mathrm{T}$. At the $300.1 \mathrm{MHz}$, (the metasurface of SRRs), the field is less homogenous with a standard deviation of
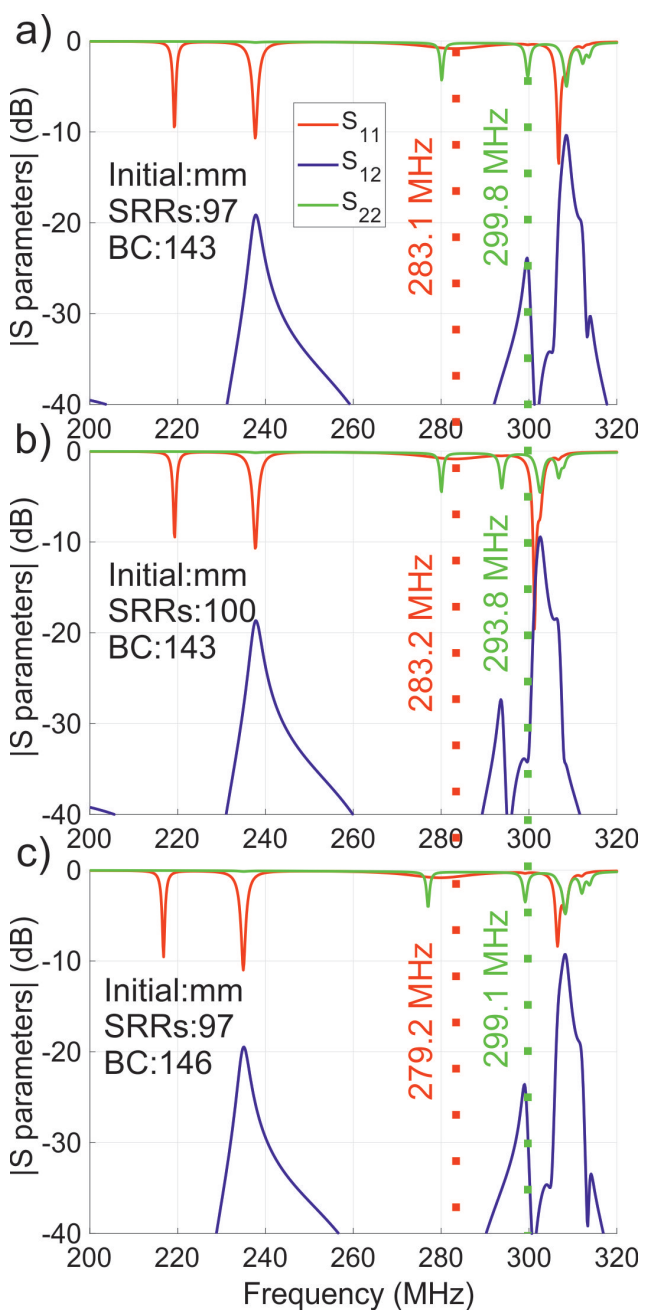

Figure 6: Study of independent tuning: (a) initial S-parameters of the dual-tuned coil; (b) length of the metasurface of SRRs have been changed; (c) length of the birdcage-like metasurface have been changed.

$0.32 \mu T$. However, this value is still sufficient for MRI preclinical application. This heterogeneity of the field can be explained by the interaction between two structures and by the presence of the phantom.

\section{Conclusion}

In this work we have proposed the new design of the dual-tuned volume coil based on closed 1D metasurfaces. We have shown a numerical study of the proposed geometry with a Finite Element Method solver. The homogeneity of $B_{1}^{+}$field at the two se- 


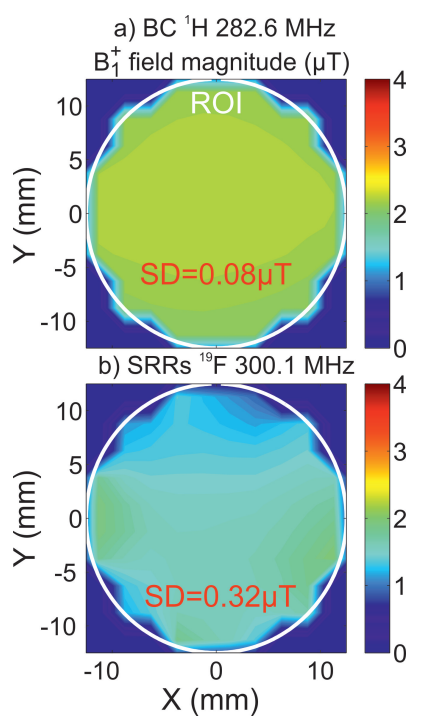

Figure 7: Magnitude of $B_{1}^{+}$at $z=0$ inside the ROI (phantom): (a) the birdcage-like metasurface; (b) the metasurface of SRRs.

lected Larmor frequencies is enough for preclinical imaging. Also, a good matching has been achieved with a high isolation between two ports. We are currently making a prototype to validate this coil experimentally.

\section{ACKNOWLEDGEMENTS}

This project has received funding from the European Unions Horizon 2020 research and innovation program under grant agreement No 736937.

\section{REFERENCES}

[1] Srinivas, M., Heerschap, A., Ahrens, E. T., Figdor, C. G., and de Vries, I. J. M., 19F MRI for quantitative in vivo cell tracking. Trends in biotechnology, 28(7), pp. 363-370, (2010).

[2] C. Jouvaud, R. Abdeddaim, B. Larrat and J. de Rosny, Volume coil based on hybridized resonators for magnetic resonance imaging, $A p$ plied Physics Letters, vol. 108, p. 023503, (2016).

[3] A. Hurshkainen, A. Nikulin, E. Georget, B. Larrat, D. Berrahou, L. Neves, P. Sabouroux, S. Enoch, I. Melchakova, P. Belov, S. Glybovski, and R. Abdeddaim, A Novel MetamaterialInspired RF-coil for Preclinical Dual-Nuclei MRI. Scientific Reports, vol 8, 9190, (2018).
[4] A. Hurshkainen, A. Nikulin, S. Glybovski, R. Abdeddaim, C. Vilmen, S. Enoch, I. Melchakova, P. Belov and D. Bendahan, A metamaterial-inspired MR antenna independently tunable at two frequencies. Metamaterials, 11th International Conf, pp. 115-117, (2017).

[5] J. Thomas Vaughan, John R. Griffiths, "RF Coils for MRI", John Wiley and Sons Ltd, (2012).

[6] Hayes, C. E., Edelstein, W. A., Schenck, J. F., Mueller, O. M. and Eash, M., An Efficient, Highly Homogeneous Radiofrequency Coil for Whole-Body NMR Imaging at 1.5 T. J. Magn. Reson. , 63, 622-628 (1985).

[7] S.B. Glybovski, A.V. Shchelokova, A.V. Kozachenko, A.P. Slobozhanyuk, I.V. Melchakova, P.A. Belov, A.V. Sokolov, A.Yu. Efimtsev and V.A. Fokin, Capacitively-loaded metasurfaces and their application in magnetic resonance imaging. 2015 Radio and Antenna Days of the Indian Ocean (RADIO), (2015).

[8] L. Solymar, E. Shamonina, "Waves in MetamaterialsI", Oxford University Press, (2009).

[9] Georget E., Luong M., Vignaud A., Giacomini E., Chazel E., Ferrand G., Amadon A., Mauconduit F., Enoch S., Tayeb G., Bonod N., Poupon C., Abdeddaim R., Stacked magnetic resonators for MRI RF coils decoupling. J. Magn. Reson., 275, 11-18 (2017). 